D I A L O U E S

IN MUSIC THERAPY

EDUCATION

\title{
ARTICLE
}

\section{Sanctuary Model Supervision: Reflections and Implications for the Music Therapy Profession}

${ }^{1}$ Kailey Campbell, MA, MT-BC*

${ }^{2}$ Alex Peuser, MM, MT-BC*

${ }^{1}$ Truman Medical Centers/University Health, Kansas City, Missouri

${ }^{2}$ Adler University, Chicago, Illinois

There are no conflicts of interest to disclose.

*These authors contributed equally to this work.

Correspondence concerning this article may be addressed to either ${ }^{1}$ kailey.mtbc@gmail.com or²apeuser15@gmail.com

Publisher: Department of Music and Arts Technology, Indiana University-Purdue University Indianapolis

Copyright: 2022 The author(s). Works published in Dialogues in Music Therapy Education are licensed using a Creative Commons Attribution 4.0 International License 


\section{Acknowledgments}

The authors would like to extend their utmost gratitude to CharCarol Fisher, MA, MTBC, MAADC II, our supervisor during internship. She has continued to be our mentor, colleague, and an influence on our careers as graduate students, professional music therapists, and researchers. Without her modeling and influence as a supervisor, this work would not have been possible.

\section{Abstract}

This paper outlines The Sanctuary Model ${ }^{\circledR}$ of supervision from the perspective of two former interns who successfully completed their music therapy internships at a Sanctuary Certified® residential psychiatric treatment facility for children and adolescents in the Midwest. The writers reflect on their experiences with supervision within this model as both supervisees and board-certified music therapists. Strengths of this model such as creating a common language with which to process clinical phenomena; formation of better, more equitable interpersonal relationships between the supervisee and supervisor; and the emphasis on parallel processes are all unique aspects of this model. Discussions of Sanctuary Model® supervision's value in music therapy clinical supervision are included.

Keywords: Supervision, Sanctuary Model, Trauma-Informed Supervision, Internship

Campbell, K., \& Peuser, A. Dialogues in Music Therapy Education,

Publisher: Department of Music and Arts Technology, Indiana University-Purdue University Indianapolis

Copyright: 2022. The author(s). Works published in Dialogues in Music Therapy Education are licensed using a Creative Commons Attribution 4.0 International License 


\section{Introduction}

\section{History and Development of The Sanctuary Model ${ }^{\circledR}$}

The Sanctuary Institute describes The Sanctuary Model囚 as "a blueprint for clinical and organizational change which, at its core, promotes safety and recovery from adversity through the active creation of a trauma-informed community" (n.d.). This model began to take shape in the 1980s in an adult inpatient psychiatric setting, where the treatment team recognized that many of their patients experienced some form of trauma (Bloom, 2017). The model itself draws upon the concept of therapeutic communities developed in the United Kingdom during World War II to treat posttraumatic stress in soldiers. Bloom and colleagues sought to create a restorative healing culture within their psychiatric setting based on the structure of therapeutic communities (Esaki et al., 2013; Whiteley, 2004). In fostering this healing culture, Bloom and colleagues wanted to avoid what Silver (1986) referred to as "sanctuary trauma," defined as expecting a safe and protective environment only to be met with more trauma (Esaki et al., 2013). Researchers hypothesized that retraumatization in treatment settings occurred when care staff and organizational leaders met service users with an authoritarian role instead of validation and respect in the wake of their service users' trauma histories (Bloom, 2017).

As the Sanctuary Model $\circledast$ evolved into its current iteration, Bloom and colleagues emphasized sensitivity to and understanding of the impact of trauma on the brain and body - tenets of trauma-informed care (Bloom, 2017). Through adaptation and continued development, care teams have implemented the model in diverse settings for service users of a range of ages and behavioral health needs (The Sanctuary Institute, 
2017). The focus of the Sanctuary Model ${ }^{\circledR}$ is on organizational change that touches and influences every level of the care structure, including those who provide direct and indirect care (Bloom, 2017; The Sanctuary Institute, 2017). Participating agencies undergo staff training and organizational restructuring, envisioning the future of their agency and the values they wish to uphold (Esaki et al., 2013; The Sanctuary Institute, 2017). The following section explores how Sanctuary-certified agencies commit to synthesizing the knowledge of multiple theoretical positions; abiding by Seven Commitments; and exploring the aspects of SELF (safety, emotion management, loss, and future). These elements relate to treatment and healing for service users, interactions among staff, and the organization's relationship with the community.

\section{About the Model}

The Sanctuary Model $\circledast$ is the synthesis of four fundamental theories: trauma theory, social learning theory, non-violent practice, and complexity theory (Abramovitz \& Bloom, 2003; Clarke, 2013). This model situates care in the context of safety, community, and recovery while focusing on the relationships between the individual or individuals receiving services, the therapist and staff, and the organization as a whole (Clarke, 2013). When implementing the Sanctuary Model®, all individuals involved work to meet Seven Commitments: a) Growth and Change, b) Open Communication, c) Democracy, d) Non-violence, e) Emotional Intelligence, f) Social Learning, and g) Social Responsibility (Abramovitz \& Bloom, 2003). These commitments are measured and explored by both the staff and service user (Bloom, 2005; Clarke, 2013). These elements inform supervision, team meetings, self-care practices, and safety guidelines (Abramovitz \& Bloom, 2003; Clarke, 2013). 


\section{The Value of The Seven Commitments within Music Therapy Supervision}

The Seven Commitments of Sanctuary were clear and present in our supervisor's approach to supervision. In this way, Sanctuary Model ${ }^{\circledR}$ supervision rejects an authoritarian approach to supervision, opting for a collaborative and democratic model. Sanctuary Model ${ }^{\circledR}$ supervision prompts increased transparency and disclosure from both supervisor and supervisee; however, in our experiences, our supervisor embodied the act of fostering safety and creating an equitable environment for us to feel supported in sharing personal details. In our work, personal disclosure allowed a glimpse into our psychological worlds, where our thoughts and emotions had a direct impact on the way we conducted ourselves in our work with service users. Reciprocity on the part of our supervisor meant that communication was equally open and transparent in both directions. Open communication meant that we could collaborate with our supervisor as problems arose. Becoming comfortable with a more personal, introspective supervision model required fostering a sense of safety within the supervisory relationship from the outset. Building foundational safety and support within the supervisory relationship can include devoting enough time to orientation, preparing supervisees for workplace challenges, and setting a regular, uninterrupted time for supervision where the lines of communication are open (Farnan, 2001; Feiner, 2019).

\section{The SELF Model}

Within the Sanctuary Model, there are four domains to recovery: a) safety, b) emotion management, c) loss, and d) future, which form the acronym for the SELF Model (Bloom, 2017). The SELF Model is a non-linear framework that creates a shared 
language to discuss and frame assessment, treatment, psychoeducation, and organizational and professional growth and change (Bloom, 2017).

\section{Safety}

The SELF Model delineates four different types of safety, including physical, psychological, social, and moral safety (Bloom, 2017). Establishing these types of safety is integral for growth (Bloom, 2017). Physical safety is defined as being safe and healthy in the outside world. Psychological safety is defined as being safe and healthy within oneself. Social safety involves the ability to be a part of a group and have one's voice heard. Moral safety is the state of being safe in a space that respects the rights of all.

\section{Emotion Management}

Emotion management and emotional intelligence are the focus of the second point of SELF (Bloom, 2017). The SELF Model affirms that it is everyone's responsibility to recognize, respond to, and manage their emotions in ways that do not compromise the safety of oneself or others (Crossnore School and Children's Home, 2017; Yanosy et al., 2009). Emotion management begins with naming one's feelings. Once one identifies their emotion, they may begin drawing upon internal or external regulation strategies to manage it effectively. Emotion management also involves recognizing the impact of one's actions on others, which relates directly to one of the Seven Commitments of the Sanctuary Mode|®: Emotional Intelligence (The Sanctuary Institute, 2011). Emotional intelligence allows individuals to understand that one's lived experiences influences their emotions and external behaviors.

\section{Loss}


Identifying and understanding loss is next within this model. Bloom (2017) notes that each change is a loss, even if this change is positive. For example, getting a new job may result in better pay and career growth opportunities, but one may lose the relationships created at the old job. Within the Sanctuary Model $\AA^{\circledR}$, those experiencing loss must address and process the emotions associated with the loss for growth to occur (Bloom, 2017).

\section{Future}

The future is the final portion of the SELF Model. Future is conceptualized through an imaginative and moral lens while emphasizing the importance of establishing safety, identifying and understanding the associated emotions, and acknowledging the pending change (Bloom, 2017).

The SELF Model is not only used with service users; care staff and their respective organizations use the SELF Model to navigate organizational habits, the creation of new policies and procedures, influence decision-making, and provide clinical supervision (Bloom, 2017).

\section{The Value of the SELF Model in Music Therapy Supervision}

As the supervisor builds and maintains the foundation of safety, issues of emotion management, loss, and future arise as a natural part of the supervisee's growth and development. Issues of emotion management occur in all stages of the internship. At the beginning of the internship, the supervisee may feel hesitant and uncertain, and experience a great deal of pressure to be more than they are (Feiner, 2019). Similiar emotion management issues arise for the supervisor, even experienced supervisors, in the context of a new supervisory relationship. The supervisor may feel pressure to 
always do the right thing; further, they may realize the complexity of supervisory issues and feel overwhelmed (Stoltenberg \& Delworth, 1987). Both supervisor and supervisee move through these emotions as the relationship deepens and they gain experience in their respective roles. Each party gains confidence, competence, and begins to better embody their identity as a professional (Stoltenberg \& Delworth, 1987; Watkins, 1993).

Perspectives on loss and future tend to surface as the internship closes. Loss is also often a part of the therapeutic process throughout the entire internship as clients leave, are discharged, or services are otherwise terminated. As the intern closes out their time at the site, they may be simultaneously navigating closure with service users and planning for future professional endeavors. Meanwhile, the supervisor may focus on making sure the intern is prepared to function independently as a professional. At this time in the supervisory relationship, the parties may have established a more collegial relationship (Farnan, 2001; Feiner, 2019). Both supervisor and supervisee experience distinct but parallel shifts in their elements of SELF over the course of the internship. The value of SELF in Sanctuary Model $®$ supervision lies in the ability for all parties in a supervisory relationship to understand each other's perspective and work collaboratively toward solutions to problems.

\section{From Theory to Practice}

\section{Team and Community Meetings}

Staff members and service users all participate in some form of consultation regarding the therapeutic process. This may happen in staff meetings or when service users check in with one another and staff (Bloom, 2017; Clarke, 2013). Community meetings are a regular part of the facility schedule and generally occur at the beginning 
of other formal gatherings such as team meetings, supervision, and therapeutic groups (Clarke, 2013). These meetings aim to ensure a consistent evaluation of safety within the organization (Bloom, 2017). Community meetings generally consist of three questions (Clarke, 2013):

1. How are you feeling?

2. What are your goals (for today or this session)?

3. Who can you ask for help?

These questions may be modified or adapted to meet the needs of the group. We, the authors, completed our music therapy internship at a facility where Community Meetings preceded any sort of group or team gathering, including group and individual music therapy sessions with service users.

\section{Psychoeducation}

Psychoeducation is present for service users and staff when working in a Sanctuary Model $\circledast$ organization (Bloom, 2017). The Sanctuary Model $®$ views "moral misalignment" as a cause of organizational and vicarious trauma (Bloom, 2017). Moral misalignment occurs under circumstances of recurrent stress leading to the justification of morally unsound actions and behavior (Bloom, 2017). Psychoeducation creates a common language for clinical staff and service users. Additionally, psychoeducation may allow open conversations for staff and service users to realign to the organization's mission and principles (Bloom, 2017). We took part in psychoeducation as a part of organizational onboarding and facilitated psychoeducation within our groups as interns. The psychoeducation delivered to the service user must align with the organization's values to create consistency and avoid communication breakdowns (Bloom, 2005). 
Staff and service users participating in psychoeducation is an example of a parallel process present throughout the Sanctuary Model®.

\section{Self-Care Plan}

Staff and service users identify self-care plans that may help them better manage the effects of trauma, vicarious or experienced (Clarke, 2013). Staff and service users are reminded to use these self-care plans proactively to reduce the impact of trauma on them. A therapist or staff member may also help a service user create their own selfcare plan and regularly check in with them on their use of the plan (Clarke, 2013; The Sanctuary Institute, n.d.). It was our experience that supervisors were also aware of some items on our self-care plans. Our supervisor would periodically remind us to use these items, reinforcing the model's safety culture.

\section{Safety Plans}

Safety plans, unlike self-care plans, are intended to be used in times where one feels overwhelmed (Child and Parent Resource Institute, n.d.). These feelings of being overwhelmed can result from a real or perceived threat to one's physical, psychological, social, or moral safety. Safety plans comprise five personalized strategies meant to be implemented when one feels unsafe (Clarke, 2013). In our experience, all individuals at our site, including staff and service users, created safety plans, and were encouraged to wear them or keep them close by as a reminder of ways to cope with stress. Figure 1 contains our safety plans from our time in internship. Photographs of our original safety plans are included in supplemental material. 


\section{Figure 1}

The authors' safety plans
1) Breathe deeply
2) Take a walk alone
3) Listen to relaxing music
4) Reach out to a friend
5) Write it down

1) Stop, Think, Analyze, \& Reframe

2) Rock

3) Mindful

4) Walk/Run

5) Write it down

6) Walk away

\section{Supervision and The Sanctuary Mode ${ }^{\circledR}$}

Practitioners within trauma-informed models like Sanctuary are called to bear witness to the traumatic narratives of their service users. These traumatic narratives disrupt the sense of social and moral order in the service user's life, as well as causing psychological and sometimes physical harm. A more holistic approach to supervision, one that broadens the scope of the treatment team to include more than just the therapist, takes place when supervision is implemented through a Sanctuary lens (Bloom et al., 2013). This model of reciprocal supervision serves as a reminder of how everyone may be involved in the reenactment of trauma and pushes individuals to actively resist this retraumatization through rescripting these situations (Bloom et al., 2013). Further, Sanctuary supervision implements the Seven Commitments, four pillars of Sanctuary, and the SELF Model, in a parallel process between the supervisee and supervisor (Bloom et al., 2013).

\section{Personal Reflections on The Sanctuary Model® as a Music Therapy Intern}

Supervision within the Sanctuary Model ${ }^{\circledR}$ primes the parties in a supervisory relationship to understand both therapeutic relationships with clients and the supervisee's internal experiences through a trauma-informed lens. Our supervisory 
experiences influenced our work with service users, our internal responses to our work, and our professional conduct throughout internship and beyond. Generally, our supervision sessions followed this basic structure:

1. Community Meeting (How are you feeling? What are your goals for this supervision? Who can help you achieve them?)

2. Completion of a SELF Model worksheet. This worksheet was explicitly created by the organization. The worksheet allowed participants to represent each aspect of SELF visually. This worksheet was used weekly and could be used to visualize the general internship experience or more specific interactions that the intern experienced.

a. Discussion of the SELF Model worksheet as it related to the goals set for supervision

3. Closing thoughts and charges for the subsequent week.

We summarize the ways in which the Sanctuary Model® of supervision influenced us in five key points, which we discuss in further detail:

1. The Sanctuary Model $\AA^{\circledR}$ provided a framework for understanding clinical music therapy work through a trauma-informed lens.

2. Participating in psychoeducation, clinical work, and supervision within the Sanctuary Model $\circledast$ illuminated internal experiences that had not been explored.

a. Participation in these elements of Sanctuary provided areas for selfexpression through personal therapy.

3. The Sanctuary Model® illuminates parallel processes between therapist and service user, as well as supervisor and supervisee. 
4. The Sanctuary Model® allowed the supervisee to disclose information at their own pace.

\section{Point 1:}

Understanding the role of music therapy at this site required constant examination of ourselves, our motives, our skills and deficits, and the holistic needs of service users through a trauma-informed lens. Bloom et al. (2013) writes:

"Mechanistic approaches to trauma healing may significantly reduce symptoms such as chronic hyperarousal, flashbacks and nightmares. For some people, symptom reduction may be enough to enable them to reconstitute a world that has made sense to them...But for others, particularly those who have had multiple experiences of betrayed trust, loss of safety and disrupted attachment, symptom relief alone is likely to be insufficient" (p. 128).

Our music therapy training leading up to internship equipped us to conceptualize music therapy treatment in a manner that emphasized physical, psychological, and social wellbeing for service users. As we entered a trauma-informed work environment, we began to learn how traumatic experiences impacted the service user's view of their life worlds, and the focus of our work broadened from that of symptom reduction to restoring a better relationship between the service user and their individual life worlds. Participation in psychoeducation and clinical supervision continuously reinforced this framework as we engaged in music therapy work with service users.

\section{Point 2:}

The Sanctuary Model's reciprocal process creates a space where internal experiences must be acknowledged to mitigate risk to the self and the service user. This 
model does not require these experiences to be divulged to the supervisor, but the SELF Model as well as the culture of safety emphasized in Sanctuary Certified sites work as the catalysts for this type of introspection. Bloom et al. (2013) notes that within the Sanctuary Model $\AA^{\circledR}$ individuals are pushed to move out of the bystander role. Therefore, both supervisor and supervisee are tasked with being curious about each other and each person's experiences. Whether or not their experiences are brought into supervision is left to the individual, but the door is always open. The curiosity displayed by the supervisor often led me to take note of my own thoughts and feelings to reflect upon in therapy and bring back to the supervisory relationship after being better processed through with a psychologist.

\section{Point 3:}

Parallel process is one of the theoretical underpinnings of the Sanctuary Model®. The Sanctuary Institute (2017) posits that an organization's operations may often parallel the traumatic symptoms in the individuals the organization serves. Therefore, noticing when these parallel processes occur is the impetus for aligning the values of the organization with the values of quality trauma-informed care. Just as care staff educate service users on the tenets of SELF and expect them to abide by the Seven Commitments of Sanctuary, these elements guide the organization's leadership in decision making. Our weekly supervisory experiences (i.e., community meeting, completion of the SELF worksheet, discussion of SELF, and goals for the following week) often paralleled the structure of music therapy sessions with service users. As interns, we took part in the processes we asked of our service users. 
Similarly, the experiences of parties within the supervisory relationship can be considered parallel processes; additionally, the supervisory relationship unfolding paralleled the unfolding of therapeutic alliances with service users. Just as therapeutic alliances unfold and deepen over time, the supervisory relationship does as well. Feiner (2019) describes three developmental phases of the supervisory relationship: 1) the "creation of space"; 2) "structure building"; 3) "reciprocity and well-being" (pp. 168-171). During the creation of space, Feiner (2019) recommends music making during supervision, as it fosters connection, allows for more profound emotional expression, and illustrates parallels between supervision and clinical work. Supervisors can effectively model holding space for service users by maintaining welcoming space for their supervisees during music making experiences.

\section{Point 4:}

The Seven Commitments were consistently employed during supervision. Open communication and non-violence were notable themes. The culture of non-violence encouraged the interns to disclose various pieces of their identities at their own pace in the early stages of building the supervisory relationship. In later iterations of supervision, open communication allowed the supervisor to ask the interns to honestly reflect on their strengths and weaknesses. This required situational awareness from both the supervisor and the supervisees. This awareness was supported by everyone within the supervisory relationship aligning to the SELF Model and using the model as a compass as opposed to a checklist (Bloom et al., 2013). This provided a more concrete set of standards with which to align. The goal of implementing the SELF Model was to 
experience safety and emotional intelligence while acknowledging losses and planning for our futures.

\section{Professional Reflections on Sanctuary Model® Supervision as Board-Certified Music Therapists}

As we transitioned from internship to professional music therapy practice, we found that elements of Sanctuary Model® supervision served us in our future workplaces.

\section{Kailey's Experiences}

Most recently, I served as a graduate teaching assistant for a music therapy program at a large university, where I was trained to understand the supervisory relationship through the lens of a developmental model. As I entered the clinical supervisor role, my experiences of supervision during internship - specifically the way my supervisor interacted with me - served as models for my supervision style. I found myself wanting to understand how my students' work impacted them and how their lived experiences might have impacted their work with service users. This practice became increasingly imperative as the COVID-19 pandemic affected the world.

Anecdotally, the COVID-19 pandemic was - and at the time of writing this, continues to be - a significant blow to our collective personal, educational, and professional lives. I saw firsthand how each of my students experienced the early days of the pandemic in different ways. Because of the disruption to the status quo, I realized how my sense of safety, emotion management, experience of loss, and view of the future were disrupted. I suspected that my students might be experiencing their own disruptions to SELF. During our weekly supervision meetings, I began asking my 
students informal questions about their elements of SELF. Upon retrospection, the students and I entered this conversation somewhat awkwardly; as mentioned in Feiner (2019), this new, more vulnerable approach to supervision might raise feelings of uncertainty in the trustworthiness of the supervisor. Acknowledging and validating these feelings of uncertainty and vulnerability within the early stages of this sort of supervision can reassure supervisees that their supervisor cares about their feelings and will prioritize creating a safe space to express those emotions.

\section{Alex's Experiences}

Since becoming board-certified I have worked in corrections, community mental health, and for a private practice. I believe that Sanctuary Supervision could have been beneficial in the first two settings. The strengths of this supervision model help create an egalitarian relationship within the supervisory relationship. Unfortunately, there was no formal framework in place for supervision within the correctional facility I worked at or within the community mental health organization $(\mathrm{CMHO})$. The $\mathrm{CMHO}$ did align itself with trauma-informed care.

Upon starting both positions I was taken aback by the lack of structure within each of my supervision sessions. They began to feel like time to check things off lists as opposed to dive into deeper work. Instead of checking things off a list, I could have spent an hour each week cultivating a deeper understanding of myself and my work through the guidance of Sanctuary and the SELF Model. I attempted to do this for myself but was met with many challenges when the culture of my workplaces did not have a similar understanding of the SELF Model. 
For me, a strength of Sanctuary Supervision was the groundwork the model laid to finding and creating safety. This groundwork would later be necessary as I navigated the challenging realities of my workplace as a minoritized therapist. The ability to ask about and visualize my safety across various domains would have helped me communicate needs to my supervisors in a more pragmatic manner which may have been perceived as less threatening to their status quo of white, cis-gender, heteronormativity. I also believe I would have been better equipped to discuss moral misalignments within my practice during supervision if the supervision I was receiving followed a structure. Earlier I noted that this model of supervision also provided me with content for my personal therapy. This was important and necessary for me, but also an area of privilege that I had access to excellent and affordable psychological services. Further, I do not think I would have had the opportunity to take time for this work without penalty to my internship hours outside of a Sanctuary Certified agency.

\section{Conclusion}

To conclude, the purpose of this paper was to discuss the elements of the trauma-informed Sanctuary Model $\AA^{\circledR}$, describe our experiences of Sanctuary Model ${ }^{\circledR}$ supervision during our music therapy internships, and discuss experienced and potential benefits to Sanctuary Model ${ }^{\circledR}$ supervision for the music therapy profession. Sanctuary Model ${ }^{\circledR}$ supervision utilizes a common language that promotes better interpersonal understanding between supervisor and supervisee. This common language is the foundation of a mutualistic, "horizontal" relationship, where both parties can collaboratively explore complex issues of therapy (Bloom et al., 2013, p. 144). The model's emphasis on parallel processes is in line with the music therapy literature on 
clinical supervision. Sanctuary Model $\AA^{\circledR}$ supervision explores several dimensions of the clinician's experience, which prompts further inquiry into the use of Sanctuary Model® supervision at different levels of clinician experience, including student, intern, and professional. 


\section{References}

Abramovitz, R., \& Bloom, S. L. (2003). Creating sanctuary in residential treatment for youth: From the "well-ordered asylum" to a "living-learning environment". Psychiatric Quarterly, 74(2), 119-135. https://doi.org/10.1023/a:1021303710275

Anglicare (n.d.). Red flag meetings [Fact sheet]. https://www.anglicare.com.au/wpcontent/uploads/2020/06/Red-Flag-PR-v2.pdf

Bloom, S. L. (2005) The Sanctuary Model of organizational change for children's residential treatment. Therapeutic Community: The International Journal for Therapeutic and Supportive Organizations, 26(1): 65-81. https://doi.org/10.1108/tc-01-2015-0005

Bloom, S. L., Yanosy, S., \& Harrison, L. C. (2013). A reciprocal supervisory network: The Sanctuary Model. In Murphy, D. \& Joseph, S. (Eds.), Trauma and the therapeutic relationship: Approaches to process and practice (pp. 126-146). Palgrave Macmillan.

Bloom, S. L. (2017). The Sanctuary Model: Through the lens of moral safety. In Gold, S. N. (Ed.), APA handbook of trauma psychology: Volume 2, trauma practice (pp. 499-513). American Psychological Association. https://doi.org/10.1037/0000020-024

Child and Parent Resource Institute (n.d.). A guide through our Sanctuary commitment [Factsheet].https://www.cpri.ca/files/8115/1206/5539/A Guide Through Our Sa nctuary Commitment.pdf

Children's Service Center (2019). Sanctuary. http://www.cscwv.org/csc/Resources/sanctuary.aspx 
Clarke, A. (2013). Sanctuary in action. Children Australia, 38(3), 95-99.

\section{https://doi.org/10.1017/cha.2013.12}

Crossnore School and Children's Home. (2017). Sanctuary reference handbook. https://www.crossnore.org/wp-content/uploads/2018/05/Sanctuary-HandbookJun-2017.pdf

Esaki, N., Benamati, J., Yanosy, S., Middleton, J. S., Hopson, L. M., Hummer, V. L., \& Bloom, S. L. (2013). The Sanctuary Model: Theoretical framework. Families in Society: The Journal of Contemporary Social Services, 94(2), 87-95.

\section{https://doi.org/10.1606/1044-3894.4287}

Farnan, L. A. (2001). Competency-based approach to intern supervision. In Forinash, M. (Ed.), Music Therapy Supervision (pp. 117-134). Barcelona Publishers. https://doi.org/10.1037/0000243-009

Feiner, S. (2019). A journey through internship supervision revisited: Roles, dynamics, and phases of the supervisory relationship. In Forinash, M. (Ed.), Music Therapy Supervision (2nd ed., pp. 157-176). Barcelona Publishers.

\section{https://doi.org/10.1037/0000243-008}

Silver, S. M. (1986). An inpatient program for post-traumatic stress disorder: Context as treatment. In Figley, C. R. (Ed.), Trauma and its wake (Vol. 2; pp. 213-231). Brunner/Mazel.

Stoltenberg, C. D. \& Delworth, U. (1987). Supervising counselors and therapists. San Francisco, CA: Jossey-Bass.

The Sanctuary Institute. (2011). The seven Sanctuary commitments [Fact sheet]. 
https://umaine.edu/socialwork/wp-content/uploads/sites/148/2017/07/Sanctuary-

Commitments.pdf

The Sanctuary Institute (2017). The Sanctuary Model.

https://www.thesanctuaryinstitute.org/about-us/the-sanctuary-model/

Watkins, C. E. (1993). Development of the psychotherapy supervisor: Concepts, assumptions, and hypotheses of the Supervisor Complexity Model. American Journal of Psychotherapy, 47(1), 58-74.

https://doi.org/10.1176/appi.psychotherapy.1993.47.1.58

Whiteley, S. (2004). The evolution of the therapeutic community. Psychiatric Quarterly, 75(3), 233-248. https://doi.org/10.1023/b:psaq.0000031794.82674.e8

Yanosy, S. M., Harrison, L. C., \& Bloom, S. L. (2009). Sanctuary staff training manual for direct care staff (2nd ed.). The Sanctuary Institute. https://nhchc.org/wpcontent/uploads/2020/01/Sanctuary-Model-Staff-Training-Manual.pdf 